\title{
A Standalone Vision Sensing System for Pseudodynamic Testing of Tuned Liquid Column Dampers
}

\author{
Kyung-Won Min, Seok-Jung Jang, and Junhee Kim \\ Department of Architectural Engineering, Dankook University, Jukjeon-dong, Suji-gu, Yongin-si, \\ Gyeonggi-do 448-701, Republic of Korea \\ Correspondence should be addressed to Junhee Kim; junheekim@dankook.ac.kr
}

Received 30 December 2015; Revised 21 March 2016; Accepted 27 March 2016

Academic Editor: Xiao-Wei Ye

Copyright (C) 2016 Kyung-Won Min et al. This is an open access article distributed under the Creative Commons Attribution License, which permits unrestricted use, distribution, and reproduction in any medium, provided the original work is properly cited.

\begin{abstract}
Experimental investigation of the tuned liquid column damper (TLCD) is a primal factory task prior to its installation at a site and is mainly undertaken by a pseudodynamic test. In this study, a noncontact standalone vision sensing system is developed to replace a series of the conventional sensors installed at the TLCD tested. The fast vision sensing system is based on binary pixel counting of the portion of images steamed in a pseudodynamic test and achieves near real-time measurements of wave height, lateral motion, and control force of the TLCD. The versatile measurements of the system are theoretically and experimentally evaluated through a wide range of lab scale dynamic tests.
\end{abstract}

\section{Introduction}

Structural vibration of tall and slender buildings induced by wind and earthquakes is one of the critical serviceability issues. Among control devices to attenuate the structural vibration, tuned liquid column damper (TLCD) has gathered much attention in both the industrial and academic fields because of its simplicity, cost-effectiveness, and easy maintenance [1]. Liquid oscillation in columns of the TLCD properly tuned to the fundamental vibration mode of the primary structure contributes to energy dissipation. The liquid oscillation exerts inertial force counteracting the structural vibration as well as damping force attributed to the coefficient head loss characteristics [2].

Majority of the previous studies regarding the TLCD are categorized into theoretical [3-6] and small-scale experimental studies [3, 7-9]. The analytical and parametrical studies are extensively conducted to confirm effects of mass ratio, structural stiffness, and geometries of the TLCD to the vibration reduction. In the studies, numerous approximate techniques have been proposed to simulate the nonlinear damping force, since exact analytical solution of the liquid motion is difficult to obtain. As a result, small-scale shaking table tests have been conducted to evaluate the TLCD especially for experimental investigation of nonlinear damping effect. However, the small-scale shaking table tests suffer still from scalability and complexity: for small-scale experiments, the nonlinearity in the similarity of a scaled model to the TLCD is issued; for a conventional shaking table test, the TLCD mounted on a complex structure needs high requirement of experimental setup [10].

The pseudodynamic test was originated by Hakuno et al. for a dynamic destructive test of a beam element partitioning a substructure of a whole structural system [11]. Since it is composed of an actuator-excited vibration experiment and an online computer simulation, the pseudodynamic test is often referred to as real-time hybrid test and many researchers developed the concept and verified the suitability of this method. In addition to its convenience compared to the traditional dynamic tests dealing with the whole structural system, investigation of nonlinear behaviors of complex structures has been addressed as one of the most important advantages of the pseudodynamic test [12]. Due to the nonlinear liquid flow in the TLCD, the pseudodynamic test has been adopted to experimentally investigate performance of the TLCD on a vibrating structure [13]. By virtue of advance of measurement 


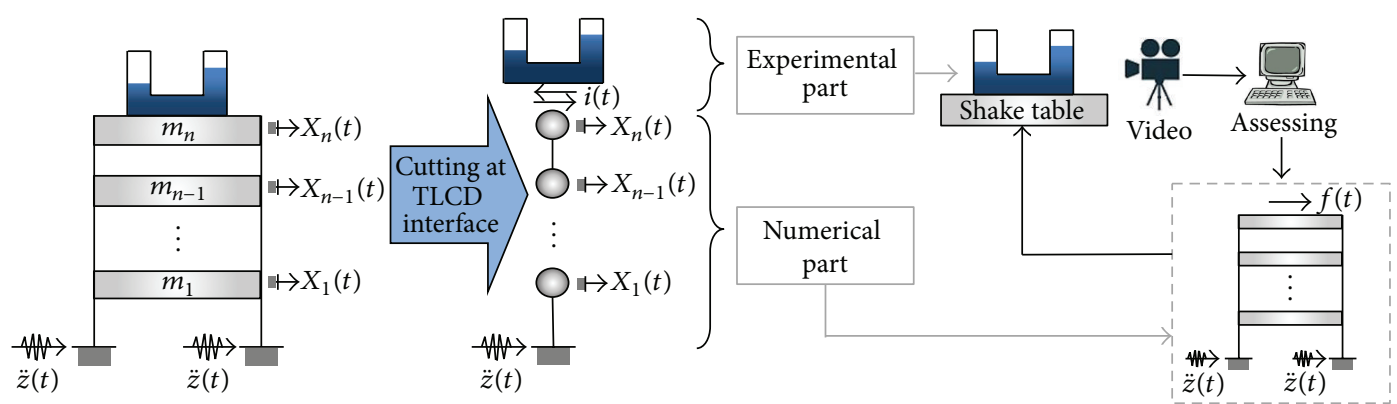

FIGURE 1: Pseudodynamic testing with a TLCD using the standalone vision sensing system.

and computer technologies, the pseudodynamic tests have been advanced and found frequently in the literature [10, $14,15]$ including authors' previous research achievements $[13,16]$.

While great advances have been made in the experimental investigation of the TLCD through the pseudodynamic test, comparatively less research has been aimed at solving the experimental complexity regarding installation of numerous tethered sensors, such as accelerometers, load cells, and wavemeters, needed to conduct the pseudodynamic test with the TLCD. Recently, there has been growing interest in noncontact vision-based sensing in the areas of structural monitoring and assessment [17-20]. A rapid, high precision, and cost-effective vision-based sensing system is preferred to the conventional sensors. When it comes to the application of the vision-based sensing to the pseudodynamic test with the TLCD, versatility of the vision-based sensing can be additionally emphasized: the aforementioned tethered sensors, for example, accelerometers, load cells, and wavemeters, needed to the test are replaced with a single vision sensor at once.

In this study, a noncontact vision sensing system is developed to facilitate execution of the pseudodynamic test with the TLCD. The system developed in this study is a standalone vision-based sensing system functioning as a simultaneous measurement of wave height, lateral motion, and control force of the TLCD. Based on the past research achievement on execution of the pseudodynamic tests, the vision-based sensing system is implemented in online pseudodynamic testing to identify dynamic characteristics of the TLCD.

\section{Vision-Based Movement Sensing of the TLCD}

The basic concept of the pseudodynamic test using a TLCD (Figure 1) is that the dynamic response is computed using the experimental result in each time step [21]. During the analysis process, a computer calculates the structural response in a time step. The whole system where the TLCD is installed on the top of the building is split into the numerical building and the experimental TLCD [22]. A shear force makes them interact at the interface. Since the shear force is exerted by the TLCD, a passive control device, it will be referred to as control force in this study. The shear force measured by a force transducer is fed back to the control computer where the building response of the story is simulated from the

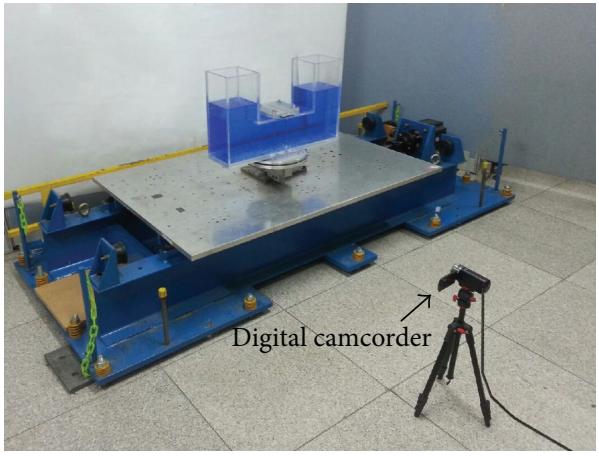

FIGURE 2: Experimental view for using image sensors.

numerical part. A vision-based sensing system is adopted to the pseudodynamic test of the TLCD.

A fast image processing technique tailored to near realtime pseudodynamic testing of the TLCD is developed for measurement of the liquid height and the lateral movement of the TLCD. A video of a laterally shaking TLCD where blue dye was added to accentuate the liquid was preliminarily taken by a digital camcorder (Figure 2). The live streaming video transferred to a computer via the USB in real time consists of a series of still frames.

The suggested technique examines a small portion of the image of each still frame specifying the still frames, termed region of interest (ROI). Two separate ROIs in the still frame are adopted for measurement of the liquid height and the lateral movement of the TLCD (Figure 3): (1) the liquid column truncated above the lower sharp-edged elbow (termed ROI 1) and (2) the lower sharp-edged elbow (termed ROI 2). Since an identical procedure is applied to both ROIs, a detailed explanation on the technique is confined only to ROI 1.

The rectangle ROI 1 is a true-color image configured as RGB information, that is, $m \times n \times 3$ data array of the three primaries in light (red, green, and blue components) for $m \times n$ pixels. In general, a graphic file format of the RGB image is 24-bit digital information, where the red, green, and blue components are 8 bits each. Thus, each component varies from 0 to 255 in a decimal number depending on its color strength. Since the liquid column of the TLCD is blue, the blue component of the pixels in the liquid column is 255 , 


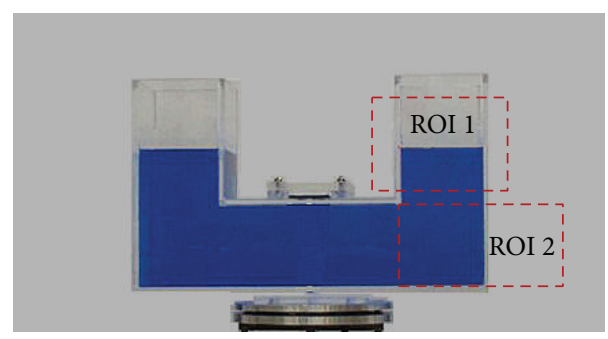

Figure 3: Two ROIs assigned in the study.

while the red and green components are trivial. On the other hand, surrounding the liquid column is pictured bright in Figure 4 due to the white screen in the background. Since white light is the sum of the three primaries in light, all of the red, green, and blue components at the pixels are nearly 255 s.

By taking components of either red or green, the truecolor image is converted to a grayscale image. In this study, red color information is exclusively selected to compose the grayscale matrix consisting of 8 bits per pixel (Figure 5). In the matrix, 256 gray colors in the pixels vary from black at the weakest intensity to white at the strongest one.

Next, the binary image is acquired from the grayscale image by designating an appropriate gray level: the 256 gray levels are grouped into two levels and each pixel is stored as a single bit, that is, 0 for black or 1 for white (Figure 6). The technique proposed in this study depends on counting of white pixels in the binary image matrix and thus accuracy is governed by quality of the binary image. Morphological operation is conducted for image enhancement: the uneven boundary becomes flat by adding white pixels to the boundaries of objects in an image.

The total sum of 1s in the binary image matrix is directly proportional to the height of the liquid column. Explanatory image matrices of the binary images processed from the aforementioned image processing are given in Figure 7 where the image matrices of the first and $i$ th frame of the streaming video are depicted, respectively.

Mapping of the total sum of the white pixels to length needs information of the number of pixels along a line of measured length. In this study, an a priori measurement of the width of the liquid column is conducted to count the number of pixels in the first frame, $B_{1}$. Thus, the height of the liquid column at the $i$ th frame is calculated as

$$
h(i)=\alpha \frac{s(i)-s(1)}{B_{1}},
$$

where $s(1)$ and $s(i)$ are the total sum of white pixels in ROI 1 at the first and $i$ th frame and $\alpha$ is the mapping factor (unit of $\mathrm{m} /$ pixel). Similarly, with the aforementioned image processing technique to measure the vertical motion of the liquid column through investigating ROI 1 , the horizontal movement of the TLCD is also measured by image processing with ROI 2 shown in Figure 3.

\section{Dynamic Equations of the TLCD on the Structure}

3.1. Equation of Motion of the TLCD. A simplified model of a U-shaped TLCD mounted on a SDOF primary structure is illustrated in Figure 8. The U-shaped tube consists of two vertical tubes and one horizontal tube and their crosssectional areas are identical, denoted as $A$ in Figure 8. When the primary structure undergoes an oscillating motion induced from the external force, $f$, the TLCD experiences the identical motion due to its firm connection to the structure. Horizontal displacement of the primary structure and vertical displacement of oscillating liquid in the TLCD are denoted as $x$ and $y$, respectively. As for the dimensions of the TLCD, horizontal and vertical column lengths are defined as $L_{h}$ and $L_{v}$, respectively.

Lagrange's equation of motion on the basis of Hamilton's principle is adopted to formulate equations of motion of the TLCD. Lagrange's equation in $y$ coordinate is given as [23]

$$
\frac{d}{d t}\left(\frac{\partial(T-V)}{\partial \dot{y}}\right)-\frac{\partial(T-V)}{\partial y}=Q_{y},
$$

where $T$ and $V$ are the kinetic and potential energies, respectively, and $Q_{y}$ represents the nonconservative force resulting from oscillating liquid in the TLCD. The three assumptions regarding simplified liquid flow, namely, (1) the sloshing behavior on the liquid surface is negligible, (2) the flow is incompressible, and (3) the dimension of the cross section is much smaller than the horizontal length of the TLCD, yield the kinetic energy of the liquid in the TLCD as

$$
T=\rho A L_{v}\left(\dot{x}^{2}+y^{2}\right)+\frac{1}{2} \rho A L_{h}(\dot{x}+\dot{y})^{2},
$$

where $\rho$ is the density of the liquid. The potential energy for restoring liquid in both vertical columns to an equilibrium level is expressed as

$$
V=-\rho A(-y) g \frac{y}{2}+\rho A y g \frac{y}{2}=\rho A g y^{2} .
$$

The nonconservative force $Q_{y}$ is written in terms of head loss coefficient $\eta$ relating to energy dissipation and velocity of liquid flow, $\dot{y}$ :

$$
Q_{y}=-\frac{1}{2} \rho A \eta|\dot{y}| \dot{y} .
$$

The equation of motion of the TLCD is derived by inserting (3), (4), and (5) into (2):

$$
m_{1} \ddot{y}+c_{1} \dot{y}+k_{1} y=-m_{2} \ddot{x},
$$

where $m_{1}=\rho A\left(L_{h}+2 L_{v}\right)=\rho A L_{e}$ is the total mass of the liquid column, $m_{2}=\rho A L_{h}$ is the mass of the horizontal liquid column, $c_{1}=(1 / 2) \rho A \eta|\dot{y}|$ is the nonlinear friction damping coefficient, and $k_{1}=2 \rho \mathrm{Ag}$ is the stiffness. Seen in (7), it is observed that the natural frequency of TLCD is

$$
\omega_{y}=\sqrt{\frac{2 g}{L_{h}+2 L_{v}}} .
$$




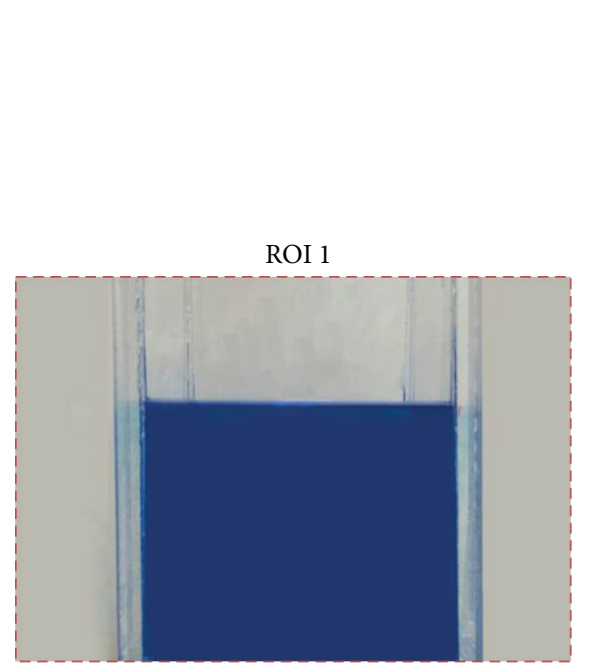

(a)

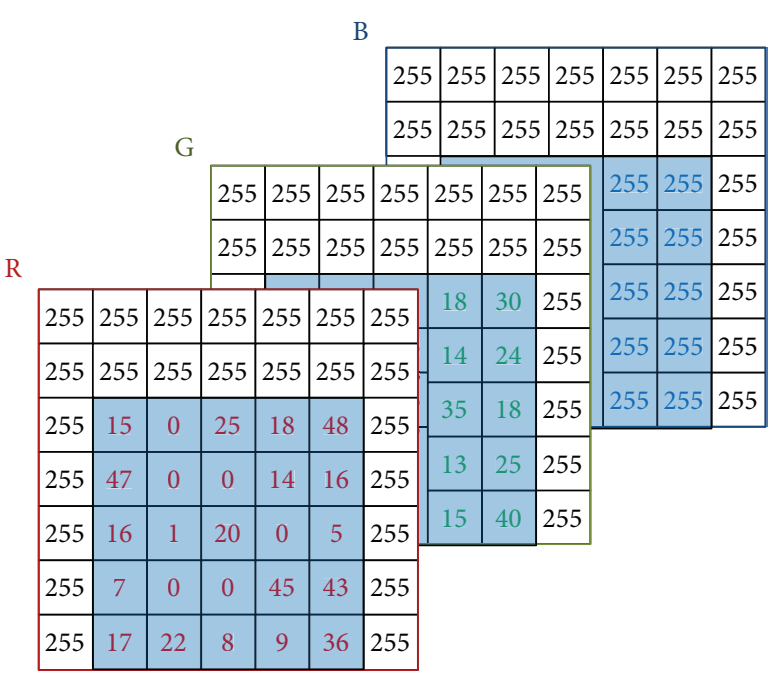

(b)

FIgURE 4: ROI 1 for wave height sensing: (a) real video image and (b) pixel example for each RGB $(m \times n \times 3)$.

ROI 1

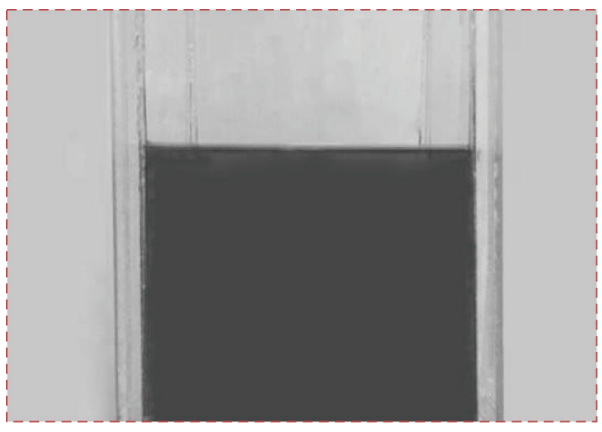

(a)

\begin{tabular}{|c|c|c|c|c|c|c|}
\multicolumn{1}{|c}{ Grayscale } \\
\hline 255 & 255 & 255 & 255 & 255 & 255 & 255 \\
\hline 255 & 255 & 255 & 255 & 255 & 255 & 255 \\
\hline 255 & 15 & 0 & 25 & 18 & 48 & 255 \\
\hline 255 & 47 & 0 & 0 & 14 & 16 & 255 \\
\hline 255 & 16 & 1 & 20 & 0 & 5 & 255 \\
\hline 255 & 7 & 0 & 0 & 45 & 43 & 255 \\
\hline 255 & 17 & 22 & 8 & 9 & 36 & 255 \\
\hline
\end{tabular}

(b)

FIGURE 5: Grayscale image: (a) real video image and (b) pixel example for each RGB $(m \times n)$.

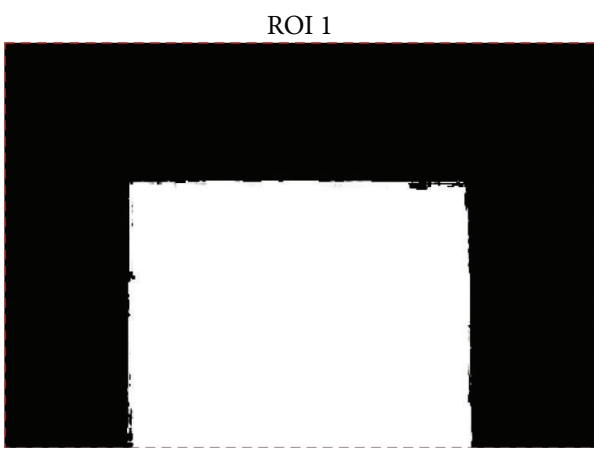

(a)

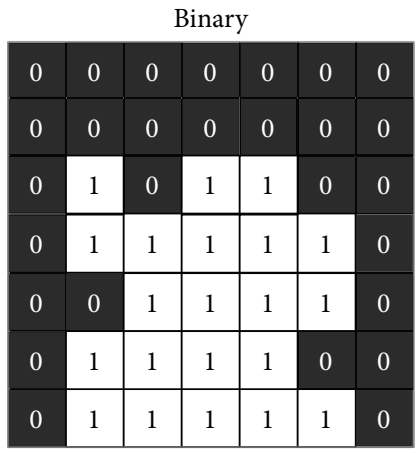

(b)

FIGURE 6: Binary image: (a) real video image and (b) information of pixel $(m \times n)$.

Considering a harmonic excitation of the primary structure with the frequency, $\omega$, and corresponding sinusoidal displacement response of the vertical liquid motion with the amplitude, $\varphi_{y}$, that is, $y=\varphi_{y} \sin \omega t$, the nonlinear friction damping can be treated as linear viscous damping by the concept of linear equivalent viscous damping:

$$
c_{\mathrm{eq}}=\frac{4}{3 \pi} \rho A \eta \varphi_{y} \omega .
$$




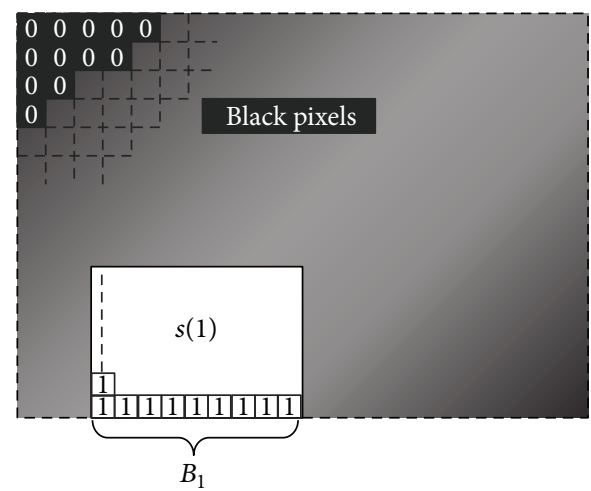

(a)

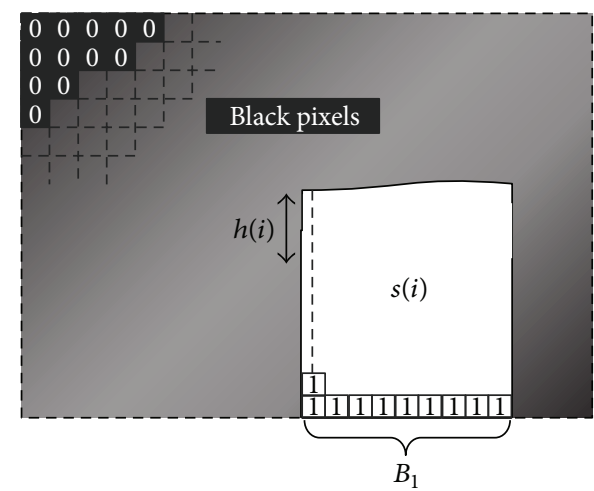

(b)

FIGURE 7: Binary information for moving liquid in ROI 1: (a) the first frame (liquid at rest) and (b) the $i$ th frame.

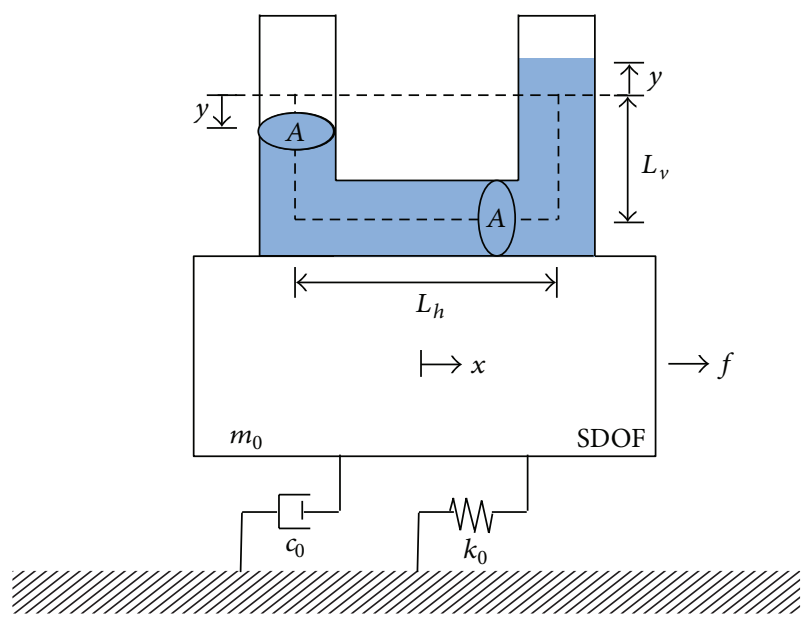

FiguRE 8: A TLCD installed to a SDOF primary structure.

Inserting (8) into the equation of motion of the TLCD yields the linearized equation of motion:

$$
m_{1} \ddot{y}+\frac{4}{3 \pi} \rho A \eta \varphi_{y} \omega \dot{y}+k_{1} y=-m_{2} \ddot{x} \text {. }
$$

Dividing both sides of (9) by $m_{1}$ and using the damping ratio lead to

$$
\ddot{y}+2 \omega_{y} \xi_{y} \dot{y}+\omega_{y}^{2} y=-p \ddot{x},
$$

where $\xi_{y}=2 \eta \varphi_{y} \beta / 3 \pi L_{e}$ is the damping ratio, $\omega_{y}=\sqrt{2 g / L_{e}}$ is the natural frequency of the TLCD, and $p=L_{h} / L_{e}$ is the ratio of the horizontal length to total length of the liquid column. The transfer function from the shake table displacement to the liquid height is obtained by taking the Fourier transform into the following:

$$
\frac{Y(i \omega)}{X(i \omega)}=\frac{p \beta^{2}}{\left(\beta^{2}-1\right)+2 \xi_{y} \beta i} \triangleq H(\beta),
$$

where $Y(i \omega)$ and $X(i \omega)$ represent the Fourier transform of the liquid height in the time domain, $y(t)$, and the shake table displacement in the time domain, $x(t)$, respectively. $\beta=$ $\omega / \omega_{y}$ is the frequency ratio of the exciting frequency to the natural frequency of the TLCD. Using Euler's identity, (11) is reformulated in the form of a complex exponential function:

$$
\begin{aligned}
H(\beta) & =\frac{p \beta^{2}}{\sqrt{\left(\beta^{2}-1\right)^{2}+\left(2 \xi_{y} \beta\right)^{2}}} \exp \left\{i \tan ^{-1} \frac{2 \xi_{y} \beta}{\left(\beta^{2}-1\right)}\right\} .
\end{aligned}
$$

As seen in (12), the transfer function, a function of the frequency ratio, is factorized into its amplitude and phase angle; that is, $H(\beta)=A(\beta) e^{i \phi(\beta)}$, where each function is defined, respectively, as follows:

$$
\begin{aligned}
& A(\beta) \triangleq \frac{p \beta^{2}}{\sqrt{\left(\beta^{2}-1\right)^{2}+\left(2 \xi_{y} \beta\right)^{2}}}, \\
& \phi(\beta) \triangleq \tan ^{-1} \frac{2 \xi_{y} \beta}{\left(\beta^{2}-1\right)} .
\end{aligned}
$$

Frequency-dependent characteristics of the transfer function are investigated analytically with respect to three different excitation frequencies: ( 1$)$ extremely low $(\beta \approx 0),(2)$ resonant $(\beta=1)$, and $(3)$ extremely high $(\beta \approx \infty)$. Firstly, liquid in the TLCD is at rest without vertical oscillation, when a very slow harmonic motion of the primary structure is applied; namely,

$$
\begin{aligned}
& \lim _{\beta \rightarrow 0} A(\beta)=0, \\
& \lim _{\beta \rightarrow 0} \phi(\beta)=0 .
\end{aligned}
$$

Secondly, damping-dependent amplitude is confirmed in the oscillating liquid motion with a phase delay of $\pi / 2$ :

$$
\begin{aligned}
& \lim _{\beta \rightarrow 1} A(\beta)=\frac{p}{2 \xi_{y}}, \\
& \lim _{\beta \rightarrow 1} \phi(\beta)=\frac{\pi}{2} .
\end{aligned}
$$


Lastly, a moderate liquid motion with an amplitude of the mass ratio, $p$, is generated from very fast primary structure's oscillation while opposing its direction against primary structure's:

$$
\begin{aligned}
& \lim _{\beta \rightarrow \infty} A(\beta)=p, \\
& \lim _{\beta \rightarrow \infty} \phi(\beta)=\pi .
\end{aligned}
$$

3.2. The Control Force of the TLCD. The control force of the TLCD to the primary structure is also derived from Lagrange's equation. Lagrange's equation in $x$ coordinate of Figure 8 is written as

$$
\frac{d}{d t}\left(\frac{\partial(T-V)}{\partial \dot{x}}\right)-\frac{\partial(T-V)}{\partial x}=f-\frac{\partial D}{\partial x},
$$

where $D$ represents the damping energy relating to the primary structure. Since the primary structure as well as the TLCD is in motion along $x$ coordinate, the kinetic energy of the primary structure equipped with the TLCD is given as

$$
T=\frac{1}{2} m_{0} \dot{x}^{2}+\rho A L_{v}\left(\dot{x}^{2}+\dot{y}^{2}\right)+\frac{1}{2} \rho A L_{h}(\dot{x}+\dot{y})^{2} .
$$

The potential energy and damping energy of the primary structure are expressed, respectively, as

$$
\begin{aligned}
& D=\frac{1}{2} c_{0} \dot{x}^{2}, \\
& V=\frac{1}{2} k_{0} x^{2} .
\end{aligned}
$$

The equation of motion of the primary structure equipped with the TLCD is derived by inserting (18) and (19) into (17):

$$
m_{0} \ddot{x}+c_{0} \dot{x}+k_{0} x=f-\left(\rho A L_{e} \ddot{x}+\rho A L_{h} \ddot{y}\right) .
$$

The right side of (20) stands for external forces acting to the primary structure. The negative forces of the last two terms are generated by liquid motion of the TLCD and consequently alleviate the total of external forces. In this study, they are termed control force, $f_{c}$, and defined as follows:

$$
f_{c} \triangleq-\left(\rho A L_{e} \ddot{x}+\rho A L_{h} \ddot{y}\right) .
$$

The transfer function from the shake table displacement to the control force is obtained by taking the Fourier transform into the following:

$$
\frac{F_{c}(i \omega)}{X(i \omega)}=m_{1} \omega^{2}+m_{1} \omega^{2} p \frac{Y(i \omega)}{X(i \omega)},
$$

where $F_{c}(i \omega)$ represents the Fourier transform of the control force in the time domain, $f_{c}(t)$. Substituting (12) into (22) and dividing both sides of (22) by $\omega^{2}$ yield

$$
\frac{F_{c}(i \omega)}{\omega^{2} X(i \omega)}=m_{1}+m_{1} p A(\beta) e^{i \phi(\beta)} .
$$

By investigating (23), frequency-dependent relationship between the control force and the shake table displacement is interpreted with respect to three different excitation frequencies: (1) extremely low $(\beta \approx 0),(2)$ resonant $(\beta=1)$, and (3) extremely high $(\beta \approx \infty)$. In the case of $\beta \approx 0, A(\beta)$ and $\phi(\beta)$ are trivial as seen in (14) and thus (23) is approximated as

$$
F_{c}(i \omega) \approx \omega^{2} m_{1} X(i \omega)
$$

This implies that the phase of the control force is close to that of the shake table displacement, since $\omega^{2}$ and $m_{1}$ are real constants. In the case of $\beta=1$, inserting (15) into (23) leads to

$$
F_{c}(i \omega) \approx \omega^{2} m_{1}\left(1+i \frac{p^{2}}{2 \xi_{y}}\right) X(i \omega) .
$$

Equation (25) implies phase delay and amplification of the control force with respect to the shake table displacement. Finally, in the case of $\beta \approx \infty$, inserting (16) into (23) leads to

$$
F_{c}(i \omega) \approx \omega^{2} m_{1}\left(1-p^{2}\right) X(i \omega)
$$

This implies that the control force and the shake table displacement are in-phase being similar to (24).

\section{Experimental Verification}

4.1. Experimental Setup. A laboratory-scaled prototype of the TLCD was fabricated with $1 \mathrm{~cm}$ thick acrylic plates. The width and height of the TLCD are 500 and $350 \mathrm{~mm}$, respectively. The inner dimension of vertical and horizontal column, that is, cross-sectional area of liquid flow, is $150 \times 150 \mathrm{~mm}$. The TLCD was mounted on a uniaxial shake table and then tap water with blue dye added was filled up to $245 \mathrm{~mm}$ from the bottom so as to set its analytical natural frequency to be $0.85 \mathrm{~Hz}\left(f_{n}=(1 / 2 \pi) \sqrt{2 g / L_{e}}=0.85 \mathrm{~Hz}\right)$. The shake table was driven by a mechanical linear actuator where rotary motion of an AC servo motor (HC-SFS502, MITSUBISHI) was converted to linear motion. The AC servo motor was operated in the velocity control mode in which angular velocity of the rotary motion is subjected to applied analog electrical signals programmed in a function generator.

A commercially available high-definition (HD) digital camcorder (HMX-QF20, SAMSUNG) was placed on a stationary tripod 3 meters away from the shake table. The digital camcorder is a $1920 \times 1080$-pixel full HD camcorder capturing 60 frames per sec. The video compression format supported by the camcorder is the standard MPEG compatible with MATLAB $^{\circledR}$. The digital camcorder was connected to a PC via the USB to transfer video files. MATLAB supports network streaming via the Microsoft ${ }^{\circledR}$ MMS protocol: the VideoFileReader object of MATLAB was used to read video frames. The streaming file transferred in real time into the PC was processed for near real-time measurement of the liquid height and the lateral movement of the TLCD by the fast vision-based method developed in this study. The figure of experimental setup with instrumentation of the vision-based sensing system is given in Figure 2. 


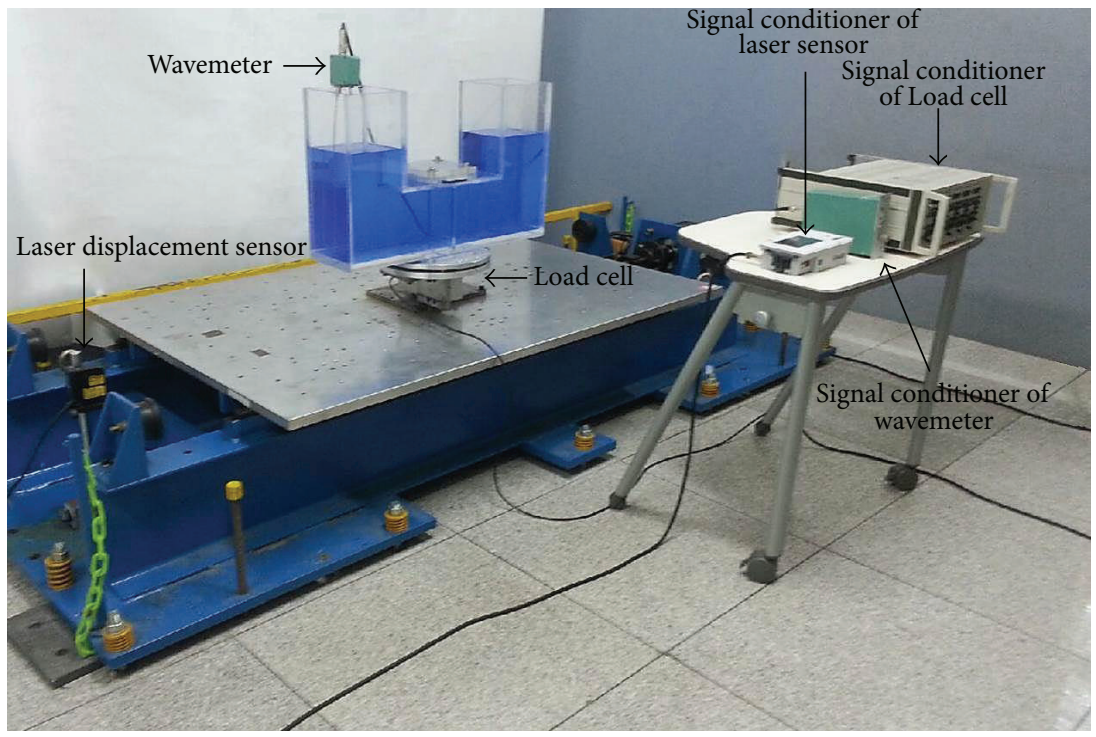

FIGURE 9: Experimental view for using conventional tethered sensors.

Accuracy of the developed measurement system was cross-checked with a series of conventional sensors: (1) the capacitive wavemeter (CH-601, KENEK) was used to measure motion of the liquid level of the TLCD. The wavemeter body was installed at the top of the TLCD while the sensor probe was immersed in the liquid column. (2) The laser displacement sensor (KL3-40, OPTEX) was utilized to measure motion of the shake table. The laser displacement sensor was set $0.85 \mathrm{~m}$ away from an edge of the shake table and sensed the shake table's displacement motion while aligning along the axis of moving direction of the shake table. (3) The strain gage load cell (OBUX-100, BONSHIN) was installed between the TLCD and the shake table to measure the base shear force referred to as control force in this study. The load cell consists of a full Wheatstone bridge configuration with four strain gages on a high strength aluminum block.

A figure of experimental setup with instrumentation of the conventional sensors is given in Figure 9. As seen, each sensor was interfaced with its own tethered signal conditioner and thus a complex configuration with costly instruments was needed to conduct the experiments. The simplicity of instrumentation of the proposed system should be noted through comparison of the proposed (Figure 2) and the conventional (Figure 9) systems.

4.2. Theoretical Verification for Harmonic Motion. Three consecutive dynamic tests with various frequencies of the harmonic excitation of the shake table were undertaken to verify the vision-based sensing system through comparison with analytical results derived in Section 3. The applied excitation frequencies are (1) 50\% of the natural frequency, that is, $0.85 \times 0.5=0.425 \mathrm{~Hz}$; (2) the natural frequency, that is, $0.85 \mathrm{~Hz}$; (3) $150 \%$ of the natural frequency, that is, $0.85 \times 1.5=1.275 \mathrm{~Hz}$. For each vibration test, $30 \mathrm{sec}$ long harmonic motion with a peak-to-peak amplitude of $\pm 7 \mathrm{~mm}$ was generated by the shake table. During the tests, the video recorded by the digital camcorder was in real time transferred to a PC for online digital image processing to yield displacements of wave height and lateral motion of the TLCD, respectively, from ROI 1 and ROI 2 (Figure 3). Since the TLCD was firmly fixed on the shake table, the lateral displacement of the shake table was identical to that of the TLCD and thus was replaced with that of the TLCD measured by the vision-based method. To compute the control force, a differential equation of (21) was used where two acceleration terms are involved. In this study, numerical differentiation was adopted to solve the differential equation of (21); accelerations were approximated as time rates of change of the slope of the displacements measured.

The vision-based methods yielded at once measurement of three physical quantities, that is, lateral displacement of the shake table, wave height in the TLCD, and control force of the TLCD during the three dynamic tests with harmonic excitation frequencies of $0.425,0.85$, and $1.275 \mathrm{~Hz}$ and the results are depicted, respectively, in Figures 10(a), 10(b), and 10 (c) where 20 to $25 \mathrm{sec}$ data are shown to investigate steady state harmonic response. Under the slow excitation of the shake table (Figure 10(a)), that is, an excitation frequency of $0.425 \mathrm{~Hz}$, little oscillation of the liquid motion is observed and thus the control force is also small as theoretically derived in (14). Furthermore, the motions of input, that is, excitation of the shake table, and output, that is, control force of the TLCD, are in-phase, which experimentally verifies (24). A notable dynamic magnification of the liquid motion of the TLCD for the resonant frequency excitation of the shake table is noticed in Figure 10(b). Significant phase differences between the shake table motion and both the liquid motion and the control force are demonstrated reflecting (15) and (25). A synchronous motion of the liquid is induced from the fast excitation of the shake table, that is, an excitation frequency of $1.275 \mathrm{~Hz}$, as seen in Figure 10 (c) where the control force poses a 180-degree out-of-phase motion to the 

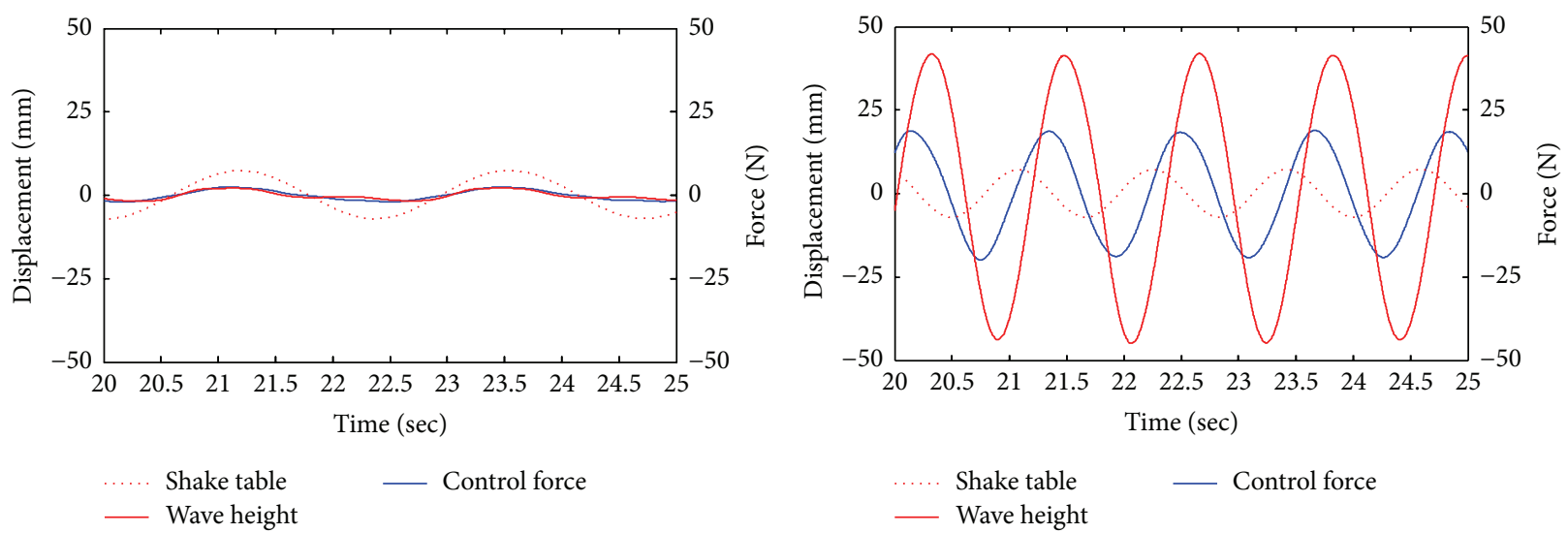

— Control force

..... Shake table

- Control force

(a)

(b)

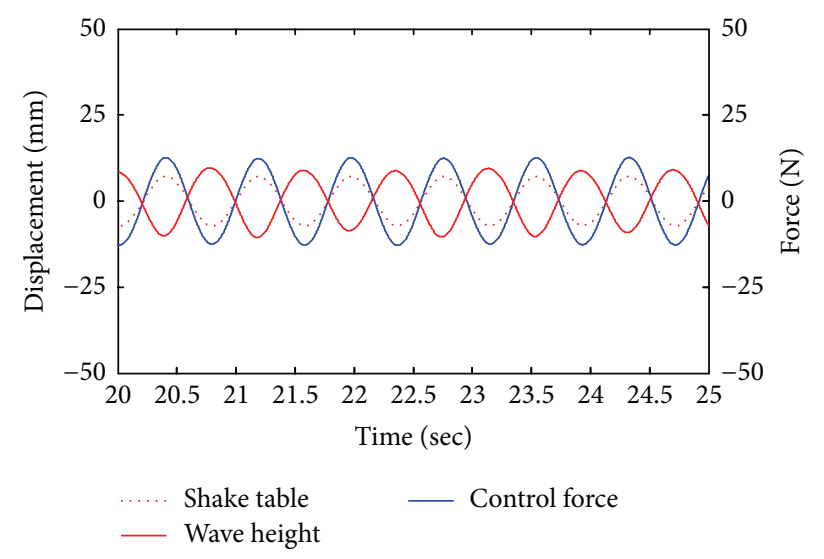

(c)

FiguRE 10: Measurements by the standalone vision sensing system for dynamic tests of harmonic excitations of (a) 0.425 , (b) 0.85 , and (c) $1.275 \mathrm{~Hz}$

shake table excitation; this experimental finding matches the analytical results of (16) and (26).

\subsection{Experimental Verification for Broadband Frequency} Motion. To verify the accuracy of the standalone vision sensing system proposed in this study, a dynamic test of the TLCD was carried out with various conventional sensors as seen in Figure 9. A wide range of frequency excitation was adopted to drive the shake table on which the TLCD was fixed. A $60 \mathrm{sec}$ long 0.2 to $2.0 \mathrm{~Hz}$ chirp signal with a constant amplitude of peak-to-peak $\pm 1 \mathrm{~V}$ was applied to excite the shake table. The real-time image processing was consecutively conducted with sequences of the freeze-frames grabbed from the streaming video for measurement of shake table motion and wave height and the results are depicted in Figures 11(a) and 11(b). For comparison purposes, time histories of measurements of the conventional sensors, that is, laser displacement meter and wavemeter, are superimposed in each figure. As seen, a very close match of two measurements is observed in each figure except the time range of 40 to $60 \mathrm{sec}$ in Figure 11(b). The notable amount of discrepancy between wave heights of the conventional and the proposed method is explained by the fact that the vision-based sensing yields the average height of the liquid column, while the wavemeter is a point sensor to measure a specific point of the liquid surface. As for a quantitative accuracy evaluation, the RMS errors were evaluated as 0.984 and $1.553 \mathrm{~mm}$, respectively, for Figures 11(a) and 11(b). The small RMS errors evaluated suggest excellent accuracy of the vision-based sensing proposed even in the wide frequency range of dynamic motion measurement.

During the dynamic test with a $60 \mathrm{sec}$ long 0.2 to $2.0 \mathrm{~Hz}$ swept-frequency excitation, the control force of the TLCD transferred to the shake table was computed by the aforementioned online numerical differentiation and depicted in Figure 12 where measurements of the load cell are superimposed for the comparison purpose. Through the whole time range of the dynamic test, an excellent agreement between two measurements of the control force is observed suggesting a very small RMS error of $0.108 \mathrm{~N}$. As a result, an excellent accuracy of the proposed vision-based control force estimation is well proved.

Comparison among experimentally identified Frequency response functions (FRFs) of the TLCD is conducted. Firstly, the FRF of the shake table displacement to the liquid height is obtained by taking the Fourier transform of measured time history data and presented in Figure 13 where the analytical FRF, that is, (11), is also superimposed for the shake of 


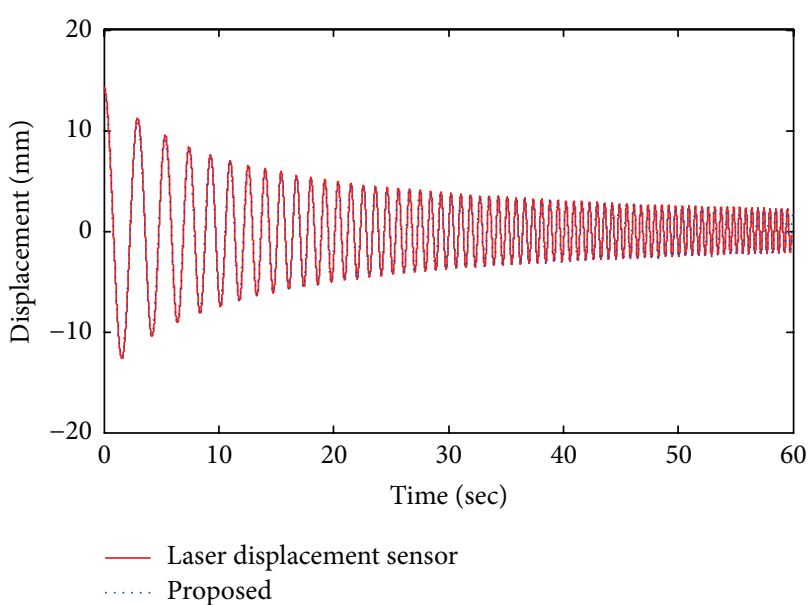

(a)

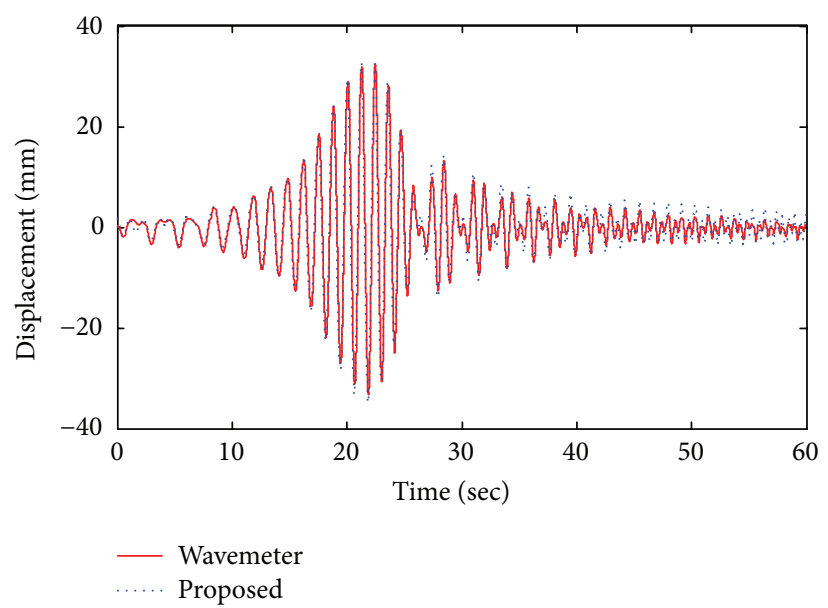

(b)

FIGURE 11: Measured dynamic motion of (a) lateral and (b) vertical motion of the TLCD.

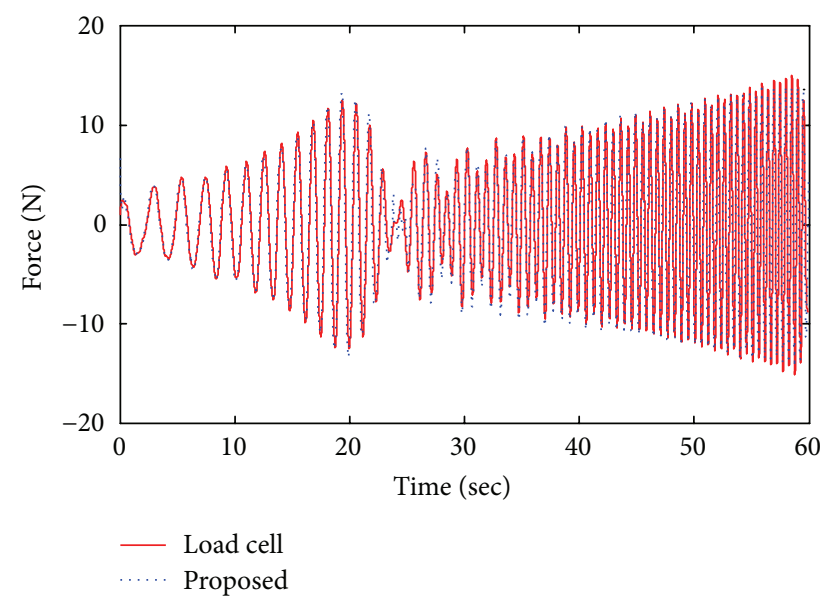

FIGURE 12: Measured control force of the TLCD.

verification. As seen in the figure, a great degree of agreement between conventional and proposed systems is confirmed in the whole range of excitation frequencies of 0.2 to $2 \mathrm{~Hz}$. Further, the analytical FRF matches the experimental FRF well. Next, the FRF from the shake table displacement to the control force is obtained and plotted in Figure 14 with the analytical FRF of (22). Again, a good degree of match among the three FRFs is verified. In addition, the natural frequency of the TLCD is clearly found as $8.24 \mathrm{~Hz}$ in the frequency domain plots. As for damping characteristics of the TLCD, head loss coefficient is experimentally calculated as 645 using the equivalent frequency-dependent damping formula derived in [8].

On the basis of the previous confirmation of the accuracy of the vision-based motion and force sensing of the TLCD, dynamic characteristics of the TLCD under a variety of harmonic motions were extensively investigated. A total of 27 harmonic excitation tests were undertaken changing the frequencies and amplitudes of the harmonic excitation of the shake table; three different peak-to-peak displacements, that

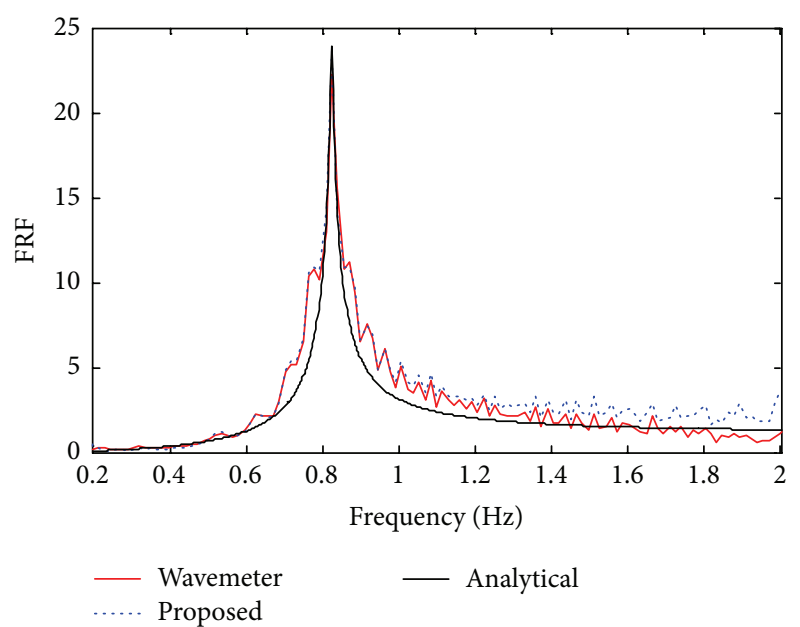

FIGURE 13: Frequency response function of lateral excitation to wave height of the TLCD.

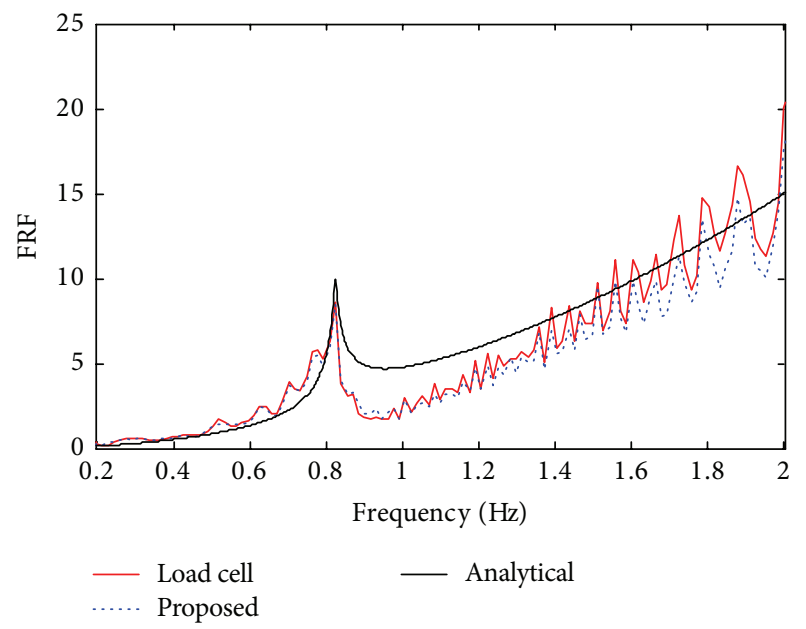

FIGURE 14: Frequency response function of lateral excitation to control force of the TLCD. 


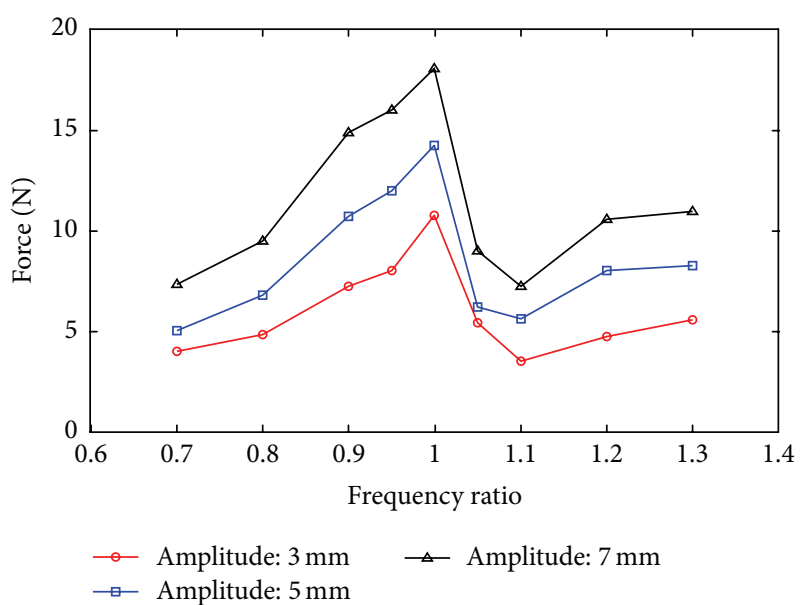

FIGURE 15: Control force of the TLCD according to change frequency ratio.

is, 3,5 , and $7 \mathrm{~mm}$, were selected as harmonic motions of the shake table and nine different excitation frequencies, that is, $0.425,0.595,0.680,0.765,0.8075,0.85,0.8925,0.935,1.02$, and $1.105 \mathrm{~Hz}$, were adopted for each frequency excitation. Figure 15 presents the control force of TLCD according to the change in the excited frequency and amplitude. The larger amplitude of the excited vibration produced the larger control force of TLCD. In the experimental result, it is evident that the tuning is greatly affected by the natural frequency of a TLCD: the control force is proportional to the excitation frequency up to the resonance and to the excitation amplitude, since the control force is the sum of motion of the shake table and liquid in the TLCD as seen in (21). However, the control forces drop next to the resonance frequency and eventually converge into specific values, since the control forces are induced from reaction force of mass media of the liquid in the horizontal tube of the TLCD. Regardless, amplitude and frequency-dependent control force exerted by the TLCD are confirmed and a rather strong nonlinearity of the control force suggests a need of the pseudodynamic test to investigate a dynamic interaction of the TLCD and the primary structure.

\section{Conclusion}

In this study, a vision sensing system is developed exclusively for dynamic characterization of tuned liquid column dampers (TLCDs) through a laboratory pseudodynamic test. The vision system developed is a standalone vision system providing various measurements of wave height, lateral motion, and control force of the TLCD. Theoretical and experimental studies are conducted to investigate control force exerted by the TLCD and demonstrate accuracy of the system. Ongoing and future work is aimed at exploring a noncontact laser device, that is, the counterpart of the vision system studied herein. Previous authors research achievements on laser-based measurement of physical quantities [24] and system identification of a vibrating structure [25] will direct the future effort.

\section{Competing Interests}

The authors declare that there are no competing interests regarding the publication of this paper.

\section{Acknowledgments}

This work is supported by the Basic Research Programs (NRF2015R1D1A1A01060643 and NRF-2015R1C1A1A01054155) of the National Research Foundation (NRF) of Korea funded by Ministry of Education, Science, and Technology (MEST).

\section{References}

[1] K.-W. Min, H.-S. Kim, S.-H. Lee, H. Kim, and S. Kyung Ahn, "Performance evaluation of tuned liquid column dampers for response control of a 76-story benchmark building," Engineering Structures, vol. 27, no. 7, pp. 1101-1112, 2005.

[2] Y. L. Xu, B. Samali, and K. C. S. Kwok, "Control of along-wind response of structures by mass and liquid dampers," Journal of Engineering Mechanics, vol. 118, no. 1, pp. 20-39, 1992.

[3] H. Gao, K. C. S. Kwok, and B. Samali, "Optimization of tuned liquid column dampers," Engineering Structures, vol. 19, no. 6, pp. 476-486, 1997.

[4] S. Colwell and B. Basu, "Experimental and theoretical investigations of equivalent viscous damping of structures with TLCD for different fluids," Journal of Structural Engineering, vol. 134, no. 1, pp. 154-163, 2008.

[5] A. Di Matteo, F. Lo Iacono, G. Navarra, and A. Pirrotta, "Direct evaluation of the equivalent linear damping for TLCD systems in random vibration for pre-design purposes," International Journal of Non-Linear Mechanics, vol. 63, pp. 19-30, 2014.

[6] F. Sakai, S. Takaeda, and T. Tamaki, "Tunes liquid column damper-new type device for suppression of building vibration," in Proceedings of the International Conference on High-Rise Building, Nanjing, China, 1989.

[7] P. A. Hitchcock, K. C. S. Kwok, R. D. Watkins, and B. Samali, "Characteristics of liquid column vibration absorbers (LCVA)I,II," Engineering Structures, vol. 19, no. 2, pp. 135-144, 1997.

[8] K.-W. Min, Y.-W. Kim, and J. Kim, "Analytical and experimental investigations on performance of tuned liquid column dampers with various orifices to wind-excited structural vibration," Journal of Wind Engineering and Industrial Aerodynamics, vol. 139, pp. 62-69, 2015.

[9] A. Di Matteo, F. Lo Iacono, G. Navarra, and A. Pirrotta, "Experimental validation of a direct pre-design formula for TLCD," Engineering Structures, vol. 75, pp. 528-538, 2014.

[10] K.-W. Min, J. Kim, and H.-R. Lee, "A design procedure of twoway liquid dampers for attenuation of wind-induced responses of tall buildings," Journal of Wind Engineering and Industrial Aerodynamics, vol. 129, pp. 22-30, 2014.

[11] M. Hakuno, M. Shidawara, and T. Hara, "Dynamic destructive test of a cantilever beam, controlled by an analog-computer," Proceedings of the Japan Society of Civil Engineers, vol. 1969, no. 171, pp. 1-9, 1969.

[12] M. Nakashima and N. Masaoka, "Real-time on-line test for MDOF systems," Earthquake Engineering and Structural Dynamics, vol. 28, no. 4, pp. 393-420, 1999.

[13] S.-K. Lee, E. C. Park, K.-W. Min, S.-H. Lee, and J.-H. Park, "Experimental implementation of a building structure with a tuned liquid column damper based on the real-time hybrid 
testing method," Journal of Mechanical Science and Technology, vol. 21, no. 6, pp. 885-890, 2007.

[14] A. A. Sorkhabi, H. Malekghasemi, and O. Mercan, "Dynamic behavior and performance evaluation of tuned liquid dampers (TLDs) using real-time hybrid simulation," in Proceedings of the ASCE Structures Congress, pp. 2153-2162, Chicago, Ill, USA, March 2012.

[15] Y. Gui, J. T. Wang, M. X. Zhou, F. Zhu, and F. Jin, "Real-time dynamic hybrid testing for multi-storey structures installed with tuned liquid damper," in Proceedings of the 6th World Conference on Structural Control and Monitoring, Barcelona, Spain, 2014.

[16] H.-N. Ho, J.-H. Lee, Y.-S. Park, and J.-J. Lee, “A synchronized multipoint vision-based system for displacement measurement of civil infrastructures," The Scientific World Journal, vol. 2012, Article ID 519146, 9 pages, 2012.

[17] J. J. Lee and M. Shinozuka, "A vision-based system for remote sensing of bridge displacement," NDT \& E International, vol. 39, no. 5, pp. 425-431, 2006.

[18] P. Olaszek, "Investigation of the dynamic characteristic of bridge structures using a computer vision method," Measurement, vol. 25, no. 3, pp. 227-236, 1999.

[19] H.-S. Choi, J.-H. Cheung, S.-H. Kim, and J.-H. Ahn, "Structural dynamic displacement vision system using digital image processing," NDT \& E International, vol. 44, no. 7, pp. 597-608, 2011.

[20] A. M. Wahbeh, J. P. Caffrey, and S. F. Masri, "A vision-based approach for the direct measurement of displacements in vibrating systems," Smart Materials and Structures, vol. 12, no. 5, pp. 785-794, 2003.

[21] F. Zhu, J. T. Wang, F. Jin, O. Altay, and T. Hara, "Real-time hybrid simulation of single and multiple tuned liquid column dampers for controlling seismic-induced response," in Proceedings of the 6th International Conference on Advances in Experimental Structural Engineering, Urbana-Champaign, Ill, USA, 2015.

[22] S.-K. Lee, E. C. Park, K.-W. Min, S.-H. Lee, L. Chung, and J.H. Park, "Real-time hybrid shaking table testing method for the performance evaluation of a tuned liquid damper controlling seismic response of building structures," Journal of Sound and Vibration, vol. 302, no. 3, pp. 596-612, 2007.

[23] J.-C. Wu, "Experimental calibration and head loss prediction of tuned liquid column damper," Tamkang Journal of Science and Engineering, vol. 8, no. 4, pp. 319-325, 2005.

[24] K. Kim and J. Kim, "Dynamic displacement measurement of a vibratory object using a terrestrial laser scanner," Measurement Science and Technology, vol. 26, no. 4, Article ID 045002, 2015.

[25] J. Kim, K. Kim, and H. Sohn, "Data-driven physical parameter estimation for lumped mass structures from a single point actuation test," Journal of Sound and Vibration, vol. 332, no. 18, pp. 4390-4402, 2013. 


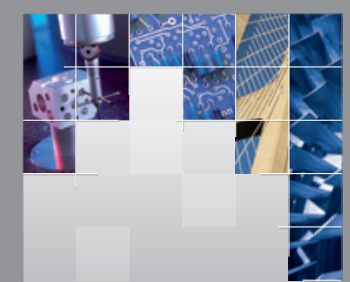

\section{Enfincering}
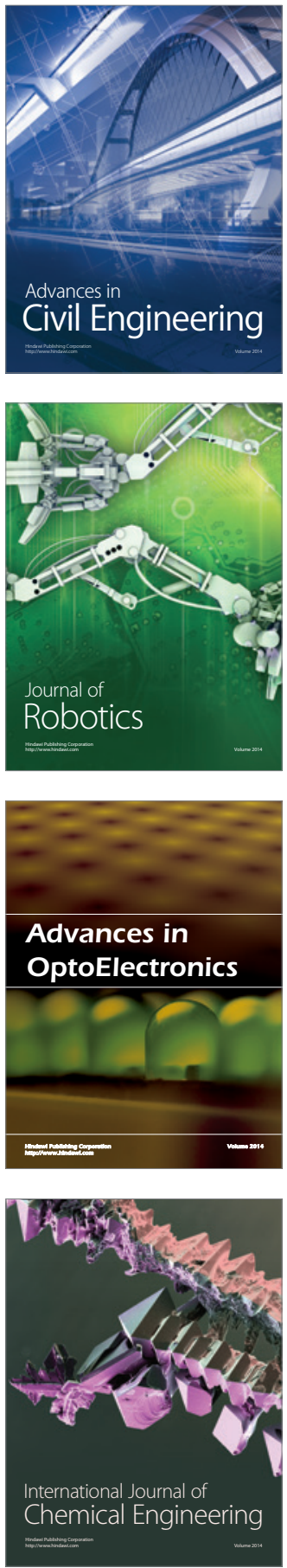

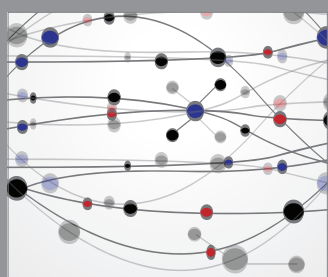

The Scientific World Journal

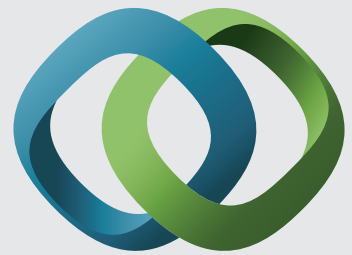

\section{Hindawi}

Submit your manuscripts at

http://www.hindawi.com
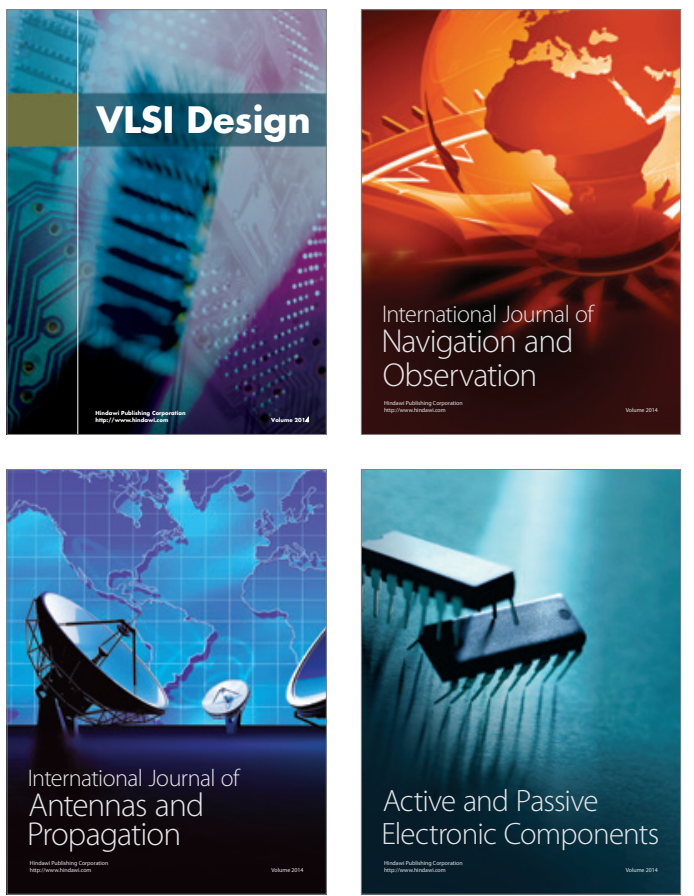
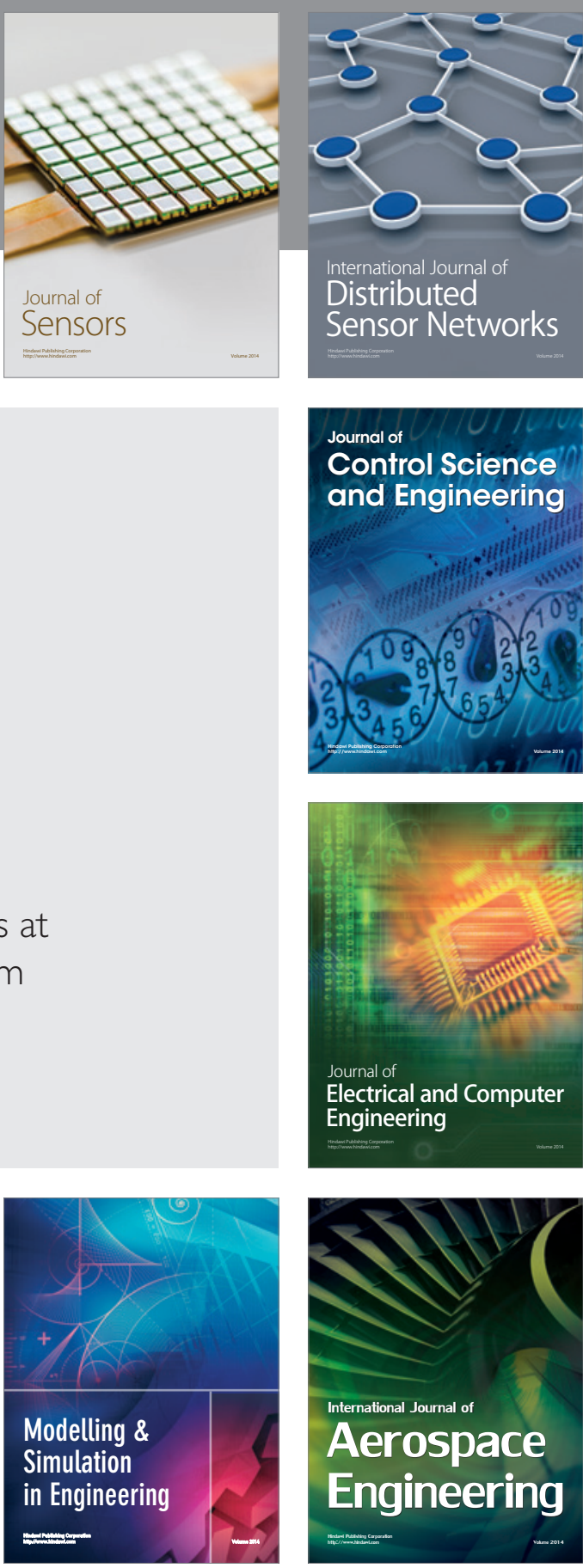

International Journal of

Distributed

Sensor Networks

Journal of

Control Science

and Engineering
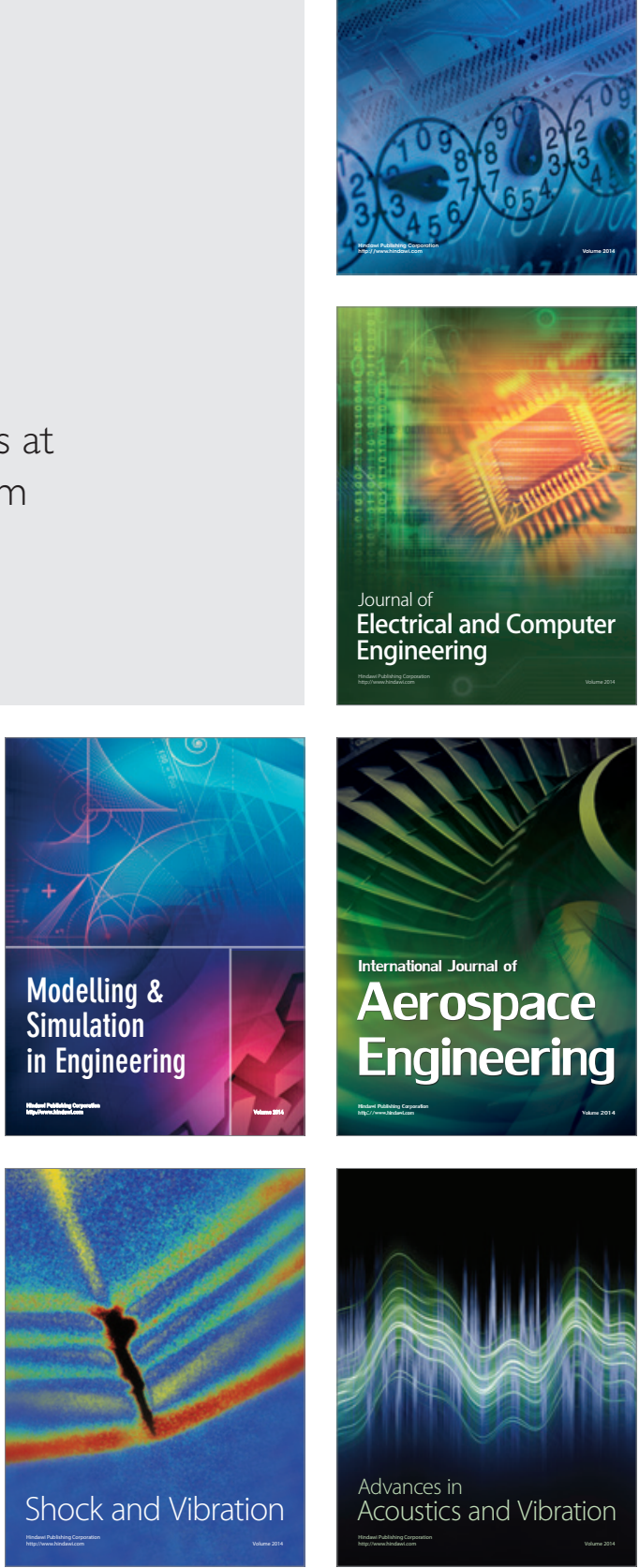